\title{
Relación entre la fuerza de agarre y pinza con dinamometría isométrica y los desórdenes músculoesqueléticos en la población adulta de Bogotá, Colombia
}

Relationship between grip and pinch force with isometric dynamometry and musculoskeletal disorders in the Bogota, Colombia adult population

Kevin Pacheco-Arias ${ }^{1}$

Pacheco-Arias, K. Relación entre la fuerza de agarre y pinza con dinamometría isométrica y los desórdenes músculoesqueléticos en la población adulta de Bogotá, Colombia. Tecnología en Marcha. Vol. 34, especial. Movilidad Estudiantil.

Octubre 2021. Pág 78-89.

doi https://doi.org/10.18845/tm.v34i5.5917 


\title{
Palabras clave
}

Fuerza; agarre; pinza; dinamometría; desórdenes musculoesqueléticos; estadística.

\section{Resumen}

El contar con entornos de trabajo saludables se ha vuelto un desafío importante para la mayoría de las empresas. Dentro de los elementos de promoción que establece la Organización Mundial de la Salud (OMS) se encuentra el adecuado diseño de los puestos de trabajo y el conocimiento de las principales limitaciones de los trabajadores. Por ello, el estudio de las habilidades físicas, como la fuerza de agarre y pinza lateral o palmar se ha vuelto un tema de interés, dado el alto índice de enfermedad laboral en la población adulta de Bogotá. El presente artículo muestra la relación estadística entre la fuerza de agarre y pinza dinamométrica, aspectos como el género, la edad y aquellas afectaciones como el dolor en el cuello, hombro, medio brazo; codo, antebrazo y dedos de los trabajadores del Instituto Colombiano de Bienestar Familiar (ICBF). Esto, mediante un estudio correlacional de corte transversal donde se estudió un N de 317 participantes; 204 mujeres y 113 hombres entre los 18-57 años. Se obtuvo como resultado que no existe relación estadística entre la fuerza de agarre con mano derecha y mano izquierda , dado que $p=0.006$ era menor al $a=0.05$. De igual forma sucedió para la fuerza de pinza lateral con mano derecha y mano izquierda, donde se obtuvo un valor de $p=0.01$, menor al $a=0.05$. Al analizar la relación entre la fuerza (agarre, pinza lateral y palmar) con respecto al género se obtuvo que existía diferencia estadística entre hombres y mujeres. Finalmente, se analizó su relación con diferentes afectaciones del sistema musculoesquelético.

\section{Keywords}

Force; grip; pinch; dynamometry; musculoskeletal disorders; statistics.

\begin{abstract}
Having a healthy work environment has become a major challenge for most companies. Within the efforts to promote safe work established by the World Healthy Organization (WHO), they are the adequate job design, the knowledge of the main limitations of the employees, therefore the study of physical capacities and abilities, such as maximum grip strength and lateral and palmar grip has become an interesting topic in consequence the high rate of occupational disease in the Bogota adult population.

This paper points out the statistical relationship between grip and pinch force and aspects such as gender, age; and neck, shoulder, middle arm, elbow, and forearm injuries suffer by the workers of the Colombian Institute of Family Welfare (ICBF). It was done throughout a crosssectional correlational study, where a 317 participants' population was included, of them: 204 women and 113 men between the ages of 18-57. As result it was obtained that there is not statistical correlation between right- and left-hand grip force, considering that the $p$ value $=0.006$ is less than the alfa value $=0.05$. Likewise, it happens with the pinch force, where the $p=0.01$ is less than the alfa value $=0.05$. When analyzing the statistical correlation between grip force and pinch (palmar and lateral) force it was obtained, as a statistical difference between men and women was obtained. Finally, it was analyzed its correlation with different disorders of the musculoskeletal system.
\end{abstract}




\section{Introducción}

La tasa de ausentismo laboral en Colombia ha venido en incremento durante los últimos años. De acuerdo con Guerrero y Díaz [1, p. 4] La Organización Internacional del Trabajo (OIT) define el ausentismo laboral como la no asistencia al trabajo por parte del empleado que desde un inicio asistiría, excluyéndose las vacaciones y huelgas. Si se ausenta por causa médica este puede definirse como el período de inasistencia laboral dada una incapacidad en el individuo, haciéndose excepción de los embarazos o temas judiciales.

Con respecto al ausentismo laboral en Colombia, según CELSA [2, p. 12] se ha encontrado que, en 2018, se presentaron 543.540 casos de los cuales la enfermedad general continúa siendo la principal causa de ausencia (63,5\%). Gran parte de estas enfermedades se encuentran asociadas con diferentes dolencias o afectaciones del sistema musculoesquelético, conocidos como desórdenes músculoesqueléticos (DME), siendo una de las patologías mayormente diagnosticadas.

De acuerdo con la Organización Mundial de la Salud [3] los desórdenes musculo esqueléticos más comunes corresponden al dolor de cuello, artrosis, dolor de espalda, las fracturas debidas a la fragilidad ósea, los traumatismos y las enfermedades inflamatorias sistémicas, como la artritis reumatoide. "Los desórdenes músculoesqueléticos son causados por trabajos fatigantes que implican posturas prolongadas, mantenidas y forzadas, con pocas posibilidades de cambio y por fuera de los ángulos confortables". [4, p. 27]

En relación con varios de los estudios realizados por el Instituto Nacional para la Seguridad y la Salud Ocupacional en el Trabajo (INSSO); en Colombia, las afectaciones del sistema musculoesquelético continúan siendo uno de los problemas de salud mayormente manifestados. Los DME "cubren una amplia gama de problemas de salud que van desde malestar, molestias y dolores menores, a condiciones médicas más graves que pueden conducir a discapacidad permanente". [5, p. 6]

Como consecuencia de estas afectaciones, la disminución en la fuerza de agarre y pinza se vuelve un punto de interés, ya que la medición de estos esfuerzos permite clínicamente determinar la severidad de las lesiones y obtener un diagnóstico en las habilidades del individuo para el desarrollo de las tareas en el trabajo y parte de sus actividades cotidianas, con el fin de evitar complicaciones futuras en la salud. "Los elementos que intervienen en el desarrollo de actividades laborales es la capacidad prensil o de agarre de las manos, la cual es una función altamente especializada que se debe a una perfecta integración motora y sensorial”. [6, p. 383] Es importante conocer los métodos de valoración funcional que permitan cuantificar la función de prensión de la mano, dado que se presenta como un indicador de riesgo de aquello que limita las funciones del individuo y que repercute en el campo laboral.

El presente artículo, tiene como fin obtener estudios recientes que permitan la valoración en la fuerza de agarre y de pinza en la población del Instituto Colombiano de Bienestar Familiar en Bogotá. Para ello, se ha realizado un muestreo preliminar con el fin de evaluar su posible asociación con los diferentes DME, la edad y el género. Al realizar esta evaluación fue conveniente considerar una serie de aspectos, como lo son: el equipo adecuado para la medición, la posición del paciente a la hora de realizar la medición, instrucciones específicas, normas estadísticas entre otros. 


\section{Metodología}

\section{Tipo de investigación y tamaño de muestra}

Este estudio consistió en una investigación correlacional de corte transversal. Se evaluó una población de 317 trabajadores del Instituto Colombiano de Bienestar Familiar (ICBF). Del total de trabajadores, 204 eran mujeres y 113 hombres, todos entre los 18 y los 57 años. Para el análisis se empleó el software estadístico Minitab 18, por medio del cual se analizaron las variables mediante estadísticos descriptivos, pruebas t-student de diferencia de medias y regresión logística binaria. Primeramente, se evaluó la relación estadística entre la fuerza de agarre, pinza lateral y palmar para mano izquierda y mano derecha. Asimismo, se analizó la relación estadística entre ambos géneros, masculino y femenino. Por último, se analizó la relación de estos esfuerzos con la edad y los desórdenes musculoesqueléticos: dolor en el cuello, hombro; medio-brazo, codo; antebrazo, muñeca y dedos.

\section{Variables de investigación}

Para el análisis fue necesario clasificar las variables a estudiar en dos grupos: Variables dependientes y variables independientes. Estas variables representan los valores categóricos y continuos que fueron empleadas a lo largo de la investigación.

Cuadro 1. Variables dependientes e independientes en el estudio.

\begin{tabular}{|c|c|c|}
\hline Variables dependientes & \multicolumn{2}{|c|}{ Variables independientes } \\
\hline Fuerza de Agarre & Dolor Cuello & Dolor Codos \\
\hline Fuerza de Pinza Palmar & Dolor Hombros & Dolor Antebrazo \\
\hline Fuerza de Pinza Lateral & Dolor Medio brazo & Edad y género \\
\hline
\end{tabular}

Fuente: Elaboración propia con información proporcionada por la USTA,2019

Herramientas y materiales para el análisis de datos

Los datos analizados fueron tomados de una base de datos suministrada por la Universidad Santo Tomás, Bogotá, Colombia. Estos datos fueron medidos por los estudiantes Cinthia Reales, Christian Zea y la docente investigadora Magda Viviana Monrroy Silva. Para el análisis se empleó el software estadístico Minitab 18, por medio del cual se analizaron las variables mediante estadísticos descriptivos, pruebas t-student de diferencia de medias y regresión logística binaria.

\section{Protocolo de medición}

Para el protocolo de medición se tomaron en cuenta cada uno de los pasos de la revisión realizada por Cinthia A. Reales, Christian R. Zea F. y Magda V. Monrroy S. [7]

1. La información se recopiló en una población de hombres y mujeres entre los 18 y 57 años pertenecientes a una empresa gubernamental.

2. Se presentó el consentimiento informado a los participantes donde conocen las consideraciones éticas, la seguridad de los datos y posturas, junto con el protocolo de medición.

3. Por medio de la dinamometría isométrica, específicamente con el dinamómetro Jamar, se valoró la fuerza máxima de pinza, pinza palmar y pinza lateral de la mano. 


\section{Resultados y discusión}

\section{Relación entre la fuerza de agarre con mano derecha y mano izquierda}

Primeramente, se analizó la relación estadística entre el esfuerzo de agarre con mano derecha y mano izquierda para la población en estudio. En el cuadro 2. Se muestra el resumen estadístico (valor mínimo, promedio, máximo, desviación estándar, etc.) de la fuerza de agarre para un $\mathrm{N}=317$.

Cuadro 2. Resumen estadístico de la fuerza máxima de agarre.

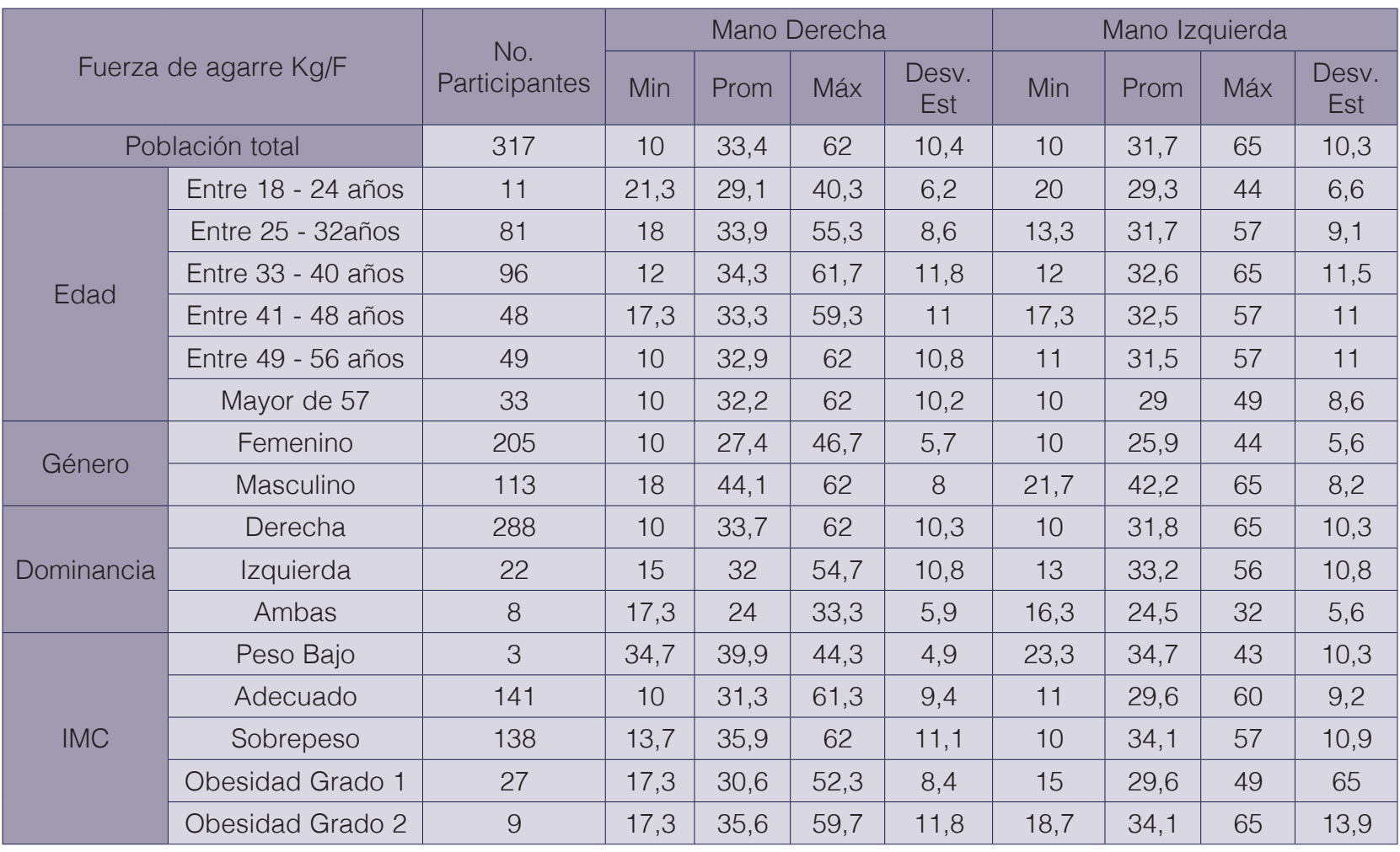

Fuente: Elaboración propia con información brindada por la USTA, 2019

De acuerdo con el cuadro 2. para la evaluación de la Fuerza de agarre con mano derecha y mano izquierda, se obtiene como resultado que en general la muestra de $\mathrm{N}=317$ presenta un mayor registro de fuerza de agarre en la mano derecha, con un promedio de 33,4 Kgf y una desviación en los datos de 10,4 Kgf. El registro más alto en edad sobre la fuerza de agarre promedio en mano derecha la registran aquellos participantes que se encuentran en un rango de 33 y 40 años con una fuerza promedio de 34,3 Kgf y una desviación de 11,8 Kgf. Para la variable de género en los participantes, se evidencia que la mayor fuerza de agarre en mano derecha la presenta el género masculino con un valor de 44,1 Kgf con una desviación de 8,0 Kgf. El valor de la fuerza de agarre de acuerdo con la mano dominante evidencia que las personas con dominancia en la mano derecha (diestros), registran mayor fuerza en su mano dominante con un promedio de 33,7 Kgf y una desviación de 10,3 Kgf. De igual forma, las personas con dominancia en la mano izquierda (zurdos), quienes registraron mayor fuerza en su mano dominante con un valor promedio de 33,2 Kgf y una desviación estándar de 10,8 Kgf. 
Finalmente, con respecto al índice, de masa corporal (IMC), el mayor registro de dicha fuerza se caracterizó en la mano derecha con los participantes catalogados con peso bajo, con un valor promedio de 39,9 Kgf con una desviación estándar de 4,9Kg/F.

Con el fin de conocer si existía diferencia significativa entre la fuerza máxima de agarre de mano derecha y mano izquierda de la población en estudio, se realizó una prueba de diferencia de medias t-student con un nivel de confianza del 95\% y un $\alpha=5 \%$, donde se planteó la siguiente hipótesis:

\section{Hipótesis}

Ho: No existe diferencia significativa entre la Fuerza de Agarre de la mano derecha y la mano izquierda.

Ho: $\mu$ agarre derecha $=\mu$ agarre izquierda.

H1: Existe diferencia significativa entre la Fuerza de Agarre de la mano derecha y la mano izquierda.

H1: $\mu$ agarre derecha $\neq \mu$ agarre izquierda.

Cuadro 3. Resultados prueba t para diferencia de medias.

\begin{tabular}{|c|c|c|c|c|c|c|c|}
\hline \multicolumn{8}{|c|}{ Prueba t student para diferencia de medias } \\
\hline \multirow{3}{*}{$\begin{array}{c}\text { Fuerza de } \\
\text { agarre }\end{array}$} & \multirow{3}{*}{$\begin{array}{c}\text { No se asumen } \\
\text { varianzas iguales }\end{array}$} & $\mathrm{t}$ & gl & valor de $p$ & $95 \%$ & $\begin{array}{l}\text { valo de } \\
\text { diferer }\end{array}$ & nza de la \\
\hline & & \multirow{2}{*}{2,74} & \multirow{2}{*}{631} & \multirow{2}{*}{0,006} & \multirow{2}{*}{2,278} & inferior & superior \\
\hline & & & & & & 0,645 & 3,912 \\
\hline
\end{tabular}

Fuente: Elaboración propia con el software Minitab 18.

Según los resultados obtenidos en el cuadro 3. es posible concluir que la fuerza de agarre con mano derecha y mano izquierda de la población adulta de Bogotá es diferente, dado que el valor de $\mathrm{p}=0,006$ es menor a $\alpha=5 \%$. Es posible concluir que se incurre en Error tipo I, al rechazar Ho.

Relación entre la fuerza máxima de pinza lateral con mano derecha y mano izquierda

En segundo lugar, se analizó la relación estadística entre la fuerza de pinza con mano derecha y mano izquierda de la población en estudio. En el cuadro 4. se muestra el estadístico descriptivo (valor mínimo, promedio, máximo, desviación estándar, etc.) para un N=317. 
Cuadro 4. Resumen estadístico fuerza máxima de pinza lateral.

\begin{tabular}{|c|c|c|c|c|c|c|c|c|c|c|}
\hline \multirow{2}{*}{\multicolumn{2}{|c|}{$\begin{array}{l}\text { Fuerza de Pinza lateral/ key } \\
\qquad(\mathrm{Kg} / \mathrm{F})\end{array}$}} & \multirow{2}{*}{$\begin{array}{c}\text { No. } \\
\text { Participantes }\end{array}$} & \multicolumn{4}{|c|}{ Mano Derecha } & \multicolumn{4}{|c|}{ Mano Izquierda } \\
\hline & & & Min & Prom & Máx & $\begin{array}{c}\text { Desv. } \\
\text { Est }\end{array}$ & Min & Prom & Máx & $\begin{array}{c}\text { Desv. } \\
\text { Est }\end{array}$ \\
\hline \multicolumn{2}{|c|}{ Población total } & 317 & 3 & 7,5 & 13,2 & 1,9 & 1,7 & 7 & 14 & 1,8 \\
\hline \multirow[t]{6}{*}{ Edad } & Entre 18 - 24 años & 11 & 5,6 & 7,1 & 9 & 1,3 & 4,8 & 6,7 & 8,8 & 1,4 \\
\hline & Entre 25 - 32años & 81 & 3,9 & 7,3 & 12 & 1,7 & 3,5 & 6,9 & 11 & 1,6 \\
\hline & Entre 33 - 40 años & 96 & 4,7 & 7,7 & 13,2 & 2 & 4,1 & 7,1 & 14 & 1,8 \\
\hline & Entre 41 - 48 años & 48 & 3,5 & 8 & 12,2 & 2,1 & 4,3 & 7,5 & 11 & 1,8 \\
\hline & Entre 49 - 56 años & 49 & 3 & 7,4 & 11,7 & 2,2 & 1,7 & 7 & 11 & 2,1 \\
\hline & Mayor de 57 & 33 & 3,5 & 7,2 & 11 & 1,8 & 3,2 & 6,4 & 9,7 & 1,5 \\
\hline \multirow[t]{2}{*}{ Género } & Femenino & 205 & 3 & 6,5 & 10,2 & 1,3 & 1,7 & 6,1 & 11 & 1,2 \\
\hline & Masculino & 113 & 5,4 & 9,4 & 13,2 & 1,5 & 4,2 & 8,7 & 14 & 1,4 \\
\hline \multirow[t]{3}{*}{ Dominancia } & Derecha & 288 & 3 & 7,6 & 12,6 & 1,9 & 1,7 & 7 & 12 & 1,7 \\
\hline & Izquierda & 22 & 4,1 & 7,3 & 13,2 & 2,5 & 3,7 & 7,4 & 14 & 2,2 \\
\hline & Ambas & 8 & 5,2 & 6,1 & 7,1 & 0,7 & 5 & 6,1 & 7,1 & 0,7 \\
\hline \multirow[t]{5}{*}{ IMC } & Peso Bajo & 3 & 8 & 9,2 & 10,5 & 1,3 & 6,5 & 7,8 & 8,4 & 1,1 \\
\hline & Adecuado & 141 & 3 & 7 & 11,5 & 1,7 & 1,7 & 6,5 & 10 & 1,5 \\
\hline & Sobrepeso & 138 & 3,5 & 8 & 12,6 & 2 & 3,2 & 7,5 & 14 & 1,9 \\
\hline & Obesidad Grado 1 & 27 & 4,8 & 7,3 & 11,4 & 1,7 & 5,1 & 7,1 & 11 & 1,6 \\
\hline & Obesidad Grado 2 & 9 & 4,6 & 8,6 & 13,2 & 2,8 & 4 & 7,6 & 10 & 2,3 \\
\hline
\end{tabular}

Fuente: Elaboración propia con información brindada por la USTA, 2019

De acuerdo con el cuadro 4 para la valoración la fuerza de pinza lateral para la mano derecha y mano izquierda es posible denotar que en general la muestra de $\mathrm{N}=317$ presenta un mayor registro de fuerza de pinza en la mano derecha, con un promedio de 7,5 Kgf y una desviación en los datos de 1,9 Kgf. El registro más alto en edad sobre la fuerza de agarre promedio en mano derecha la registran aquellos que se encuentran en un rango entre los 41 y 48 años con una fuerza promedio de 8,0 Kgf y una desviación de 2,1 Kgf. Para la variable de género en los participantes, se evidencia que la mayor fuerza de agarre en mano derecha la presenta el género masculino con un valor de 9,4 Kgf y una desviación de 13,2 Kgf. El valor de la fuerza de pinza lateral de acuerdo con la mano dominante evidencia que las personas con dominancia en la mano derecha (diestros) registran mayor fuerza en su mano dominante con un promedio de 7,6 Kgf y una desviación de 1,9 Kgf. De igual forma, las personas con dominancia en la mano izquierda (zurdos) registraron mayor fuerza en su mano dominante con un valor promedio de 7,4 Kgf y una desviación estándar de 2,2 Kgf. Con respecto al índice de masa corporal (IMC), el mayor registro de dicha fuerza se caracterizó en la mano derecha con los participantes catalogados con peso bajo, con un valor promedio de 9,2 Kgf con una desviación estándar de $1,3 \mathrm{Kg} / \mathrm{F}$.

Al igual que el caso anterior, para conocer si existía diferencia significativa entre la fuerza máxima de pinza de mano derecha y mano izquierda de la población en estudio, se realizó una prueba de diferencia de medias t-student con un nivel de confianza del $95 \%$ y un $\alpha=5 \%$, donde se planteó la siguiente hipótesis:

\section{Hipótesis}

Ho: No existe diferencia significativa entre la Fuerza de Pinza Lateral mano derecha y la mano izquierda. 
Ho: $\mu$ pinza derecha $=\mu$ pinza lateral izquierda.

H1: Existe diferencia significativa entre la Fuerza de Pinza Lateral de la mano derecha y la mano izquierda.

H1: $\mu$ pinza derecha $\neq \mu$ pinza lateral izquierda.

Cuadro 5. Resultados prueba t student para diferencia de medias.

\begin{tabular}{|c|c|c|c|c|c|c|c|}
\hline \multicolumn{7}{|c|}{ Prueba t para diferencia de medias } \\
\hline \multirow{2}{*}{$\begin{array}{c}\text { Fuerza de } \\
\text { agarre }\end{array}$} & $\begin{array}{c}\text { No se asumen } \\
\text { varianzas iguales }\end{array}$ & $\mathrm{t}$ & $\mathrm{gl}$ & valor de $\mathrm{p}$ & \multicolumn{3}{|c|}{$95 \%$ intervalo de confianza de la } \\
\cline { 6 - 9 } & & 3,45 & 627 & 0,001 & Diferencia & inferior & superior \\
\cline { 6 - 9 } & & & & 0,506 & 0,218 & 0,794 \\
\hline
\end{tabular}

Fuente: Elaboración propia con software Minitab 18.

Según los datos que se muestran en el cuadro 5. es posible concluir que la fuerza de pinza lateral con mano derecha y mano izquierda presentan diferencia significativa. Esto dado a que el valor de $p=0,001$ es menor al $\alpha=5 \%$, por lo que se rechaza Ho. Es posible inducir que se incurre en Error tipo I, al rechazar el Ho.

Luego de concluir que para los esfuerzos analizados anteriormente (agarre y pinza) tanto la mano derecha como la mano izquierda eran significativamente diferentes se procede analizar la relación estadística con respecto al género. Los resultados se resumen en el cuadro 6.

Cuadro 6. Relación estadísticas tipo de fuerza vrs género.

\begin{tabular}{|c|c|c|c|}
\hline \multicolumn{2}{|c|}{ Resultados relación estadística Fuerzas vrs Género } \\
\hline \multirow{2}{*}{ Variable Continua } & Variable Categórica & \multicolumn{2}{c|}{ Relación estadística } \\
\cline { 2 - 4 } & Derecha & Izquierda \\
\hline Fuerza Agarre & Género (Masculino vrs Femenino) & No & No \\
\hline Fuerza Pinza Lateral & Género (Masculino vrs Femenino) & No & No \\
\hline Fuerza Pinza Palmar & Género (Masculino vrs Femenino) & No & No \\
\hline
\end{tabular}

De acuerdo con el cuadro 6 es posible denotar que para cada una de las variables fuerza de agarre, pinza lateral y pinza palmar derecha e izquierda, no existe relación estadística entre el género masculino y femenino. Es decir, la fuerza de los hombres y las mujeres dista significativamente.

\section{Regresión logística binaria}

Luego de analizar si existía diferencia significativa entre el género masculino y femenino por cada una de las fuerzas presentadas, se procede analizar su relación estadística con la edad y otras afectaciones comunes como el tipo de dolor presente en el paciente (cuello, hombro; medio-brazo, codo; antebrazo, muñeca y dedos) a fin de conocer si cada una de estas variables afecta directamente la respuesta. "La regresión logística es una técnica estadística multivariable destinada al análisis de una relación de dependencia entre una variable dependiente y un conjunto de variables independientes, de forma similar a como actúa el análisis de regresión lineal clásico" [8, p. 5]. 
Relación entre la fuerza de agarre, el género, la edad y los desórdenes musculo esqueléticos (DME)

En el cuadro 7. se presentan los resultados obtenidos a partir del análisis de regresión logística binaria entre la fuerza de agarre y las variables independientes.

Cuadro 7. Resumen resultados ajuste modelo logístico binario fuerza máximo de agarre.

\begin{tabular}{|c|c|c|c|c|c|}
\hline \multicolumn{6}{|c|}{ Resultados ajuste modelo logístico binario } \\
\hline \multirow{2}{*}{ Tipo de dolor } & \multirow{2}{*}{ Variable Continua } & \multicolumn{2}{|c|}{ Relación estadística } & \multirow{2}{*}{$\begin{array}{c}\text { Variable } \\
\text { Categórica }\end{array}$} & \multirow{2}{*}{$\begin{array}{l}\text { Relación } \\
\text { estadística }\end{array}$} \\
\hline & & Derecho & Izquierdo & & \\
\hline \multirow{2}{*}{ Cuello } & Fuerza Agarre & No & No & \multirow{2}{*}{ Género } & \multirow{2}{*}{ Sí } \\
\hline & Edad & \multicolumn{2}{|c|}{ No } & & \\
\hline \multirow{2}{*}{ Hombro derecho } & Fuerza Agarre & No & No & \multirow{2}{*}{ Género } & \multirow{2}{*}{ Sí } \\
\hline & Edad & \multicolumn{2}{|c|}{ Sí } & & \\
\hline \multirow{2}{*}{ Hombro izquierdo } & Fuerza Agarre & No & No & \multirow{2}{*}{ Género } & \multirow{2}{*}{ Sí } \\
\hline & Edad & \multicolumn{2}{|c|}{ No } & & \\
\hline \multirow{2}{*}{ Medio brazo derecho } & Fuerza Agarre & No & No & \multirow{2}{*}{ Género } & \multirow{2}{*}{ No } \\
\hline & Edad & \multicolumn{2}{|c|}{ No } & & \\
\hline \multirow{2}{*}{ Medio brazo izquierdo } & Fuerza Agarre & No & No & \multirow{2}{*}{ Género } & \multirow{2}{*}{ No } \\
\hline & Edad & \multicolumn{2}{|c|}{ No } & & \\
\hline \multirow{2}{*}{ Codo derecho } & Fuerza Agarre & No & No & \multirow{2}{*}{ Género } & \multirow{2}{*}{ No } \\
\hline & Edad & \multicolumn{2}{|c|}{ No } & & \\
\hline \multirow{2}{*}{ Codo izquierdo } & Fuerza Agarre & No & No & \multirow{2}{*}{ Género } & $\mathrm{No}$ \\
\hline & Edad & & & & NNO \\
\hline Antehrazo derecho & Fuerza Agarre & No & No & Género & $\mathrm{No}$ \\
\hline Amentazo derecno & Edad & & & Gentero & NO \\
\hline Antebrazo izauierdo & Fuerza Agarre & No & No & Género & $\mathrm{No}$ \\
\hline Amenta $\angle 0$ izquierco & Edad & & & Genero & NO \\
\hline Muñeca derecha & Fuerza Agarre & No & No & Género & $\mathrm{No}$ \\
\hline ivineca derecria & Edad & & & Gerlero & INO \\
\hline Muñeca izquierda & Fuerza Agarre & No & No & Género & $\mathrm{No}$ \\
\hline & Edad & & & verter & 100 \\
\hline Dedos derecha & Fuerza Agarre & No & No & Género & No \\
\hline Deaus aerectra & Edad & & & gentero & INO \\
\hline & Fuerza Agarre & No & No & & \\
\hline Dedos izquierda & Edad & & & Genero & NO \\
\hline
\end{tabular}

Según los resultados del cuadro 7. se obtiene que, ninguna de las afectaciones o dolencias por DME afecta la fuerza de agarre(derecha o izquierda) del paciente. Sin embargo, se puede apreciar que, el dolor en el hombro derecho y los dedos sí presentan relación estadística con la edad, lo que significa que estas dolencias aumentan o disminuyen conforme la edad del paciente. Con respecto al género, se puede apreciar que, únicamente el dolor en el cuello y los hombros se presenta con la misma intensidad tanto en hombres como mujeres, pues sí presentan relación estadística significativa. 
Relación entre la fuerza de pinza lateral, el género, la edad y los desórdenes musculo esqueléticos (DME)

En el cuadro 8. se presentan los resultados obtenidos a partir del análisis de regresión logística binaria entre la fuerza de pinza lateral y las variables independientes.

Cuadro 8. Resumen resultados ajuste modelo logístico binario fuerza pinza lateral.

\begin{tabular}{|c|c|c|c|c|c|}
\hline \multicolumn{6}{|c|}{ Resultados ajuste modelo logístico binario } \\
\hline \multirow{2}{*}{ Tipo de dolor } & \multirow{2}{*}{ Variable Continua } & \multicolumn{2}{|c|}{ Relación estadística } & \multirow{2}{*}{$\begin{array}{l}\text { Variable } \\
\text { Categórica }\end{array}$} & \multirow{2}{*}{$\begin{array}{l}\text { Relación } \\
\text { estadística }\end{array}$} \\
\hline & & Derecho & Izquierdo & & \\
\hline \multirow{2}{*}{ Cuello } & Fuerza Pinza Lateral & No & No & \multirow{2}{*}{ Género } & \multirow{2}{*}{ Sí } \\
\hline & Edad & & & & \\
\hline \multirow{2}{*}{$\begin{array}{l}\text { Hombro } \\
\text { derecho }\end{array}$} & Fuerza Pinza Lateral & No & No & \multirow{2}{*}{ Género } & \multirow{2}{*}{ Sí } \\
\hline & Edad & & & & \\
\hline \multirow{2}{*}{$\begin{array}{l}\text { Hombro } \\
\text { izquierdo }\end{array}$} & Fuerza Pinza Lateral & Sí & No & \multirow{2}{*}{ Género } & \multirow{2}{*}{ Sí } \\
\hline & Edad & & & & \\
\hline \multirow{2}{*}{$\begin{array}{c}\text { Medio brazo } \\
\text { derecho }\end{array}$} & Fuerza Pinza Lateral & No & No & \multirow{2}{*}{ Género } & \multirow{2}{*}{ Sí } \\
\hline & Edad & & & & \\
\hline \multirow{2}{*}{$\begin{array}{l}\text { Medio brazo } \\
\text { izquierdo }\end{array}$} & Fuerza Pinza Lateral & No & No & \multirow{2}{*}{ Género } & \multirow{2}{*}{ No } \\
\hline & Edad & & & & \\
\hline \multirow{2}{*}{ Codo derecho } & Fuerza Pinza Lateral & Sí & No & \multirow{2}{*}{ Género } & \multirow{2}{*}{ No } \\
\hline & Edad & & & & \\
\hline \multirow{2}{*}{ Codo izquierdo } & Fuerza Pinza Lateral & No & No & \multirow{2}{*}{ Género } & \multirow{2}{*}{ No } \\
\hline & Edad & & & & \\
\hline \multirow{2}{*}{$\begin{array}{c}\text { Antebrazo } \\
\text { derecho }\end{array}$} & Fuerza Pinza Lateral & No & No & \multirow{2}{*}{ Género } & \multirow{2}{*}{ No } \\
\hline & Edad & & & & \\
\hline \multirow{2}{*}{$\begin{array}{l}\text { Antebrazo } \\
\text { izquierdo }\end{array}$} & Fuerza Pinza Lateral & No & No & \multirow{2}{*}{ Género } & $\mathrm{No}$ \\
\hline & Edad & & & & \\
\hline Muñeca & Fuerza Piza Lateral & No & No & Género & $\mathrm{No}$ \\
\hline derecha & Edad & & & & NO \\
\hline Muñeca & Fuerza Pinza Lateral & No & No & Gónorn & $\mathrm{No}$ \\
\hline izquierda & Edad & & & Gentero & TNO \\
\hline Dedos derecha & Fuerza Pinza Lateral & Sí & No & Género & No \\
\hline Dedos izquierda & Fuerza Pinza Lateral & No & No & Género & $\mathrm{N}$ \\
\hline & Edad & & & Genero & NO \\
\hline
\end{tabular}

De acuerdo con los resultados del cuadro 8. se observa que, de todas las afectaciones analizadas por DME, únicamente el dolor en hombro derecho, codo derecho y los dedos de la mano derecha afectan la fuerza de pinza lateral del paciente. A su vez, se denota que tanto el dolor en el hombro derecho como los dedos de la mano derecha se relaciona estadísticamente con la edad, por lo que estos aumentan o disminuyen en proporción a esta. Por otra parte, también se puede observar que, el dolor en el cuello, los hombros (derecho e izquierdo) y el medio brazo derecho se presenta con la misma intensidad tanto para hombres como mujeres al existir relación estadística significativa. 
Relación entre la fuerza de pinza palmar, el género, la edad y los desórdenes musculo esqueléticos (DME)

En el cuadro 9. se presentan los resultados obtenidos a partir del análisis de regresión logística binaria entre la fuerza de pinza palmar y las demás variables independientes.

Cuadro 9. Resumen resultados ajuste modelo logístico binario fuerza pinza palmar.

\begin{tabular}{|c|c|c|c|c|c|}
\hline \multicolumn{6}{|c|}{ Resultados ajuste modelo logístico binario } \\
\hline \multirow{2}{*}{ Tipo de dolor } & \multirow{2}{*}{ Variable Continua } & \multicolumn{2}{|c|}{ Relación estadística } & \multirow{2}{*}{$\begin{array}{c}\text { Variable } \\
\text { Categórica }\end{array}$} & \multirow{2}{*}{$\begin{array}{l}\text { Relación } \\
\text { estadística }\end{array}$} \\
\hline & & Derecho & Izquierdo & & \\
\hline \multirow{2}{*}{ Cuello } & Fuerza Pinza Palmar & No & No & \multirow{2}{*}{ Género } & \multirow{2}{*}{ Sí } \\
\hline & Edad & \multicolumn{2}{|c|}{ No } & & \\
\hline \multirow{2}{*}{ Hombro derecho } & Fuerza Pinza Palmar & No & No & \multirow{2}{*}{ Género } & \multirow{2}{*}{ Sí } \\
\hline & Edad & \multicolumn{2}{|c|}{ No } & & \\
\hline \multirow{2}{*}{ Hombro izquierdo } & Fuerza Pinza Palmar & No & No & \multirow{2}{*}{ Género } & \multirow{2}{*}{ Sí } \\
\hline & Edad & \multicolumn{2}{|c|}{ No } & & \\
\hline \multirow{2}{*}{$\begin{array}{l}\text { Medio brazo } \\
\text { derecho }\end{array}$} & Fuerza Pinza Palmar & Sí & No & \multirow{2}{*}{ Género } & \multirow{2}{*}{ Sí } \\
\hline & Edad & \multicolumn{2}{|c|}{ No } & & \\
\hline \multirow{2}{*}{$\begin{array}{l}\text { Medio brazo } \\
\text { izquierdo }\end{array}$} & Fuerza Pinza Palmar & No & No & \multirow{2}{*}{ Género } & \multirow{2}{*}{ No } \\
\hline & Edad & \multicolumn{2}{|c|}{ No } & & \\
\hline \multirow{2}{*}{ Codo derecho } & Fuerza Pinza Palmar & Sí & No & \multirow{2}{*}{ Género } & \multirow{2}{*}{ No } \\
\hline & Edad & \multicolumn{2}{|c|}{ No } & & \\
\hline \multirow{2}{*}{ Codo izquierdo } & Fuerza Pinza Palmar & No & No & \multirow{2}{*}{ Género } & \\
\hline & Edad & & & & No \\
\hline Antebrazo & Fuerza Pinza Palmar & No & No & & $\mathrm{No}$ \\
\hline derecho & Edad & & & Genero & NO \\
\hline Antebrazo & Fuerza Pinza Palmar & No & No & & $\mathrm{N}$ \\
\hline izquierdo & Edad & & & Genero & NO \\
\hline Muñeca derecha & Fuerza Pinza Palmar & No & No & Género & $\mathrm{Nh}$ \\
\hline Ivuneca derecna & Edad & & & Genero & NO \\
\hline Muñeca jzquierda & Fuerza Pinza Palmar & No & No & Género & $\mathrm{No}$ \\
\hline Ivineca izquieraa & Edad & & & Genero & NO \\
\hline Redpc dereshe & Fuerza Pinza Palmar & No & No & Gónoro & $\mathrm{N} / \mathrm{s}$ \\
\hline vedos derecna & Edad & & & Genero & NO \\
\hline Redes izauierda & Fuerza Pinza Palmar & No & No & Género & $\mathrm{No}$ \\
\hline Dedos izquierda & Edad & & & Genero & NO \\
\hline
\end{tabular}

De acuerdo con los resultados del cuadro 9 es posible denotar que, de todas las afectaciones analizadas, el dolor en el medio brazo y codo derechos son las únicas que presentan relación estadística con la fuerza de pinza palmar derecha. También es posible denotar que el dolor en los dedos de la mano derecha se relaciona estadísticamente con la edad, por lo que este aumenta o disminuye en proporción a esta. Por último, es posible apreciar que, el dolor en el cuello, los hombros y el medio brazo derecho es el mismo tanto para hombres como mujeres, pues presentan relación estadística significativa. 


\section{Conclusiones y/o recomendaciones}

- Se determinó que existe diferencia estadística significativa entre la fuerza de agarre con mano derecha y mano izquierda de la población en estudio, con un valor de $p=0,006$ menor al alfa de 0,05.

- Se obtiene que existe diferencia estadística significativa entre la fuerza de pinza con mano izquierda y mano derecha, con un valor de p de 0,001 menor al alfa de 0,05.

- Se determinó que existe diferencia estadística significativa entre el género: masculino y femenino con respecto a cada una de las fuerzas: agarre, pinza lateral y palmar.

- Se determinó que la fuerza de agarre no se ve afectada por ninguna de los desórdenes musculoesqueléticos en el paciente.

- Se obtuvo que la fuerza de pinza lateral se ve afectada únicamente por el dolor en el hombro, dolor en el codo derecho, dolor en los dedos derecha y la edad la edad del paciente.

- Se concluye que la fuerza de pinza palmar, únicamente se ve afectada por el dolor en el medio brazo derecho.

- Con los resultados obtenidos de esta investigación es posible dar continuidad a otros estudios relacionados con la influencia de los desórdenes musculoesqueléticos en el campo laboral y salud de la población adulta de Bogotá, Colombia.

\section{Agradecimientos}

Agradezco a los semilleros de investigación científica de la Universidad Santo Tomás de Aquino (USTA), Bogotá Colombia por permitirme realizar mi pasantía y llevar a cabo esta investigación, en especial a los docentes investigadores el Ing. Diego Fernando Sánchez Zambrano y a la Ing. Magda Viviana Monrroy Silva por su apoyo, asesoría y acompañamiento en todo momento. Finalmente agradezco a mi universidad; el Instituto Tecnológico de Costa Rica, al programa de Movilidad Estudiantil (TEC-CONARE) y la Escuela de Ingeniería en Producción Industrial por permitirme el desarrollo de experiencia tan enriquecedora en el trabajo de investigaciones como la expuesta a largo de este artículo.

\section{Referencias}

[1] A. Guerrero y K. Diaz, «Caracterización del ausentismo laboral por causas médicas en una empresa de alimentos de la ciudad de Bogotá D.C 2013-2014, „Universidad del Rosario, Bogotá, 2016.

[2] CESLA, «Tercer Informe de seguimiento sobre ausentismo laboral e incapacidades médicas, Medellín, Colombia, 2019.

[3] OMS, «Organización Mundial de la Salud,» 9 Agosto 2019. [En línea]. Available: https://www.who. int/es/news-room/fact-sheets/detail/musculoskeletal-conditions\#: : :text=Como\%20se\%20indica\%20en\%20 la. asociados\%20como\%20tendones\%20y\%20ligamentos.

[4] C. Ordónez, E. Gómez y A. Calvo , «Desórdenes músculo esqueléticos relacionados con el trabajo,» Revista Colombiana de Salud ocupacional, p. 26, 2016.

[5] S. Malca Sandoval, «Trastornos musculoesqueléticos de origen laboral en el cuello y las extremidades superiores de los fisioterapeutas en Cataluña,» 2017. [En línea]. Available: https://www.tdx.cat/bitstream/handle/10803/420862/Tsms1de1.pdf?sequence=2\&isAllowed=y. [Último acceso: 05 Septiembre 2017].

[6] C. Zea, M. P. Caro y L. A. Quintana , «LA. Análisis de la disminución de fuerza de agarre en la mano por uso de guante en actividades de aseo y cafetería., "Revista de ciencias de la salud, p. 383, 2016.

[7] M. V. Monrroy Silva, C. Zea y C. Reales, Interviewees, Relación entre la Fuerza de Agarre y Pinza con Dinamometría Isométrica y los desórdenes músculoesqueléticos en la población adulta de Bogotá. [Entrevista]. 2019.

[8] P. López Roldan y S. Fachelli, Metodología de la investigación Social Cuantitativa, Barcelona: Universidad Autónoma de Barcelona, 2015, p. 5. 